\title{
Present Situation and Research Progress of Kidney Function Recoverability Evaluation of Acute Kidney Injury Patient
}

\section{Yu-Long Yao \\ Yuan Gao}

Department of Critical Care Medicine, Renji Hospital, School of Medicine, Shanghai Jiao Tong University, Shanghai, 200127, People's Republic of China

Correspondence: Yuan Gao

Department of Critical Care Medicine, Renji Hospital, School of Medicine,

Shanghai Jiao Tong University, No. 160 of Pujian Street, Pudong New Area,

Shanghai, 200127, People's Republic of China

Tel +86 2I 58752345

Fax +862158752345

Email ylydr_09sea@163.com

\begin{abstract}
Acute kidney injury (AKI) is a critical illness in clinic. The guideline recommendation of kidney disease for improving global outcomes regards urine volume and creatinine as standards to evaluate kidney functions. However, urine volume and creatinine have a certain delay for kidney function evaluation, and these would be interfered by many factors. Whether the renal function of AKI patients can recover is very important, which affects the quality of life of patients. Therefore, the present study reviews the application situation and research progress of the recoverability evaluation of AKI patient kidney function from three aspects: conventional indexes, biomarkers, and imaging methods of kidney function.
\end{abstract}

Keywords: acute kidney injury, renal function, creatinine, urine output

\section{Introduction}

Acute kidney injury (AKI) is a common critical illness in clinic. According to a domestic research report, the 90-day fatality rate of AKI patients can reach up to $34 \%$. ${ }^{1,2}$ According to the follow-up data, it was found that patients with AKI, who have been discharged for 10 years, have a higher fatality rate. ${ }^{3}$ For surviving AKI patients, $20-50 \%$ of these patients develop chronic kidney disease, and 5\% of these patients develop end-stage kidney disease. ${ }^{4}$ AKI leads to poor prognosis, and even death. $^{5}$

The AKI diagnostic standards recommended by KDIGO are as follows: (1) kidney function suddenly decreases within 48 hours, and the absolute value of serum creatinine ( $\mathrm{SCr}$ ) increases $\geq 26.5 \mu \mathrm{mol} / \mathrm{L}(0.3 \mathrm{mg} / \mathrm{dL})$; (2) SCr increases by $\geq 1.5$ times of the base value; (3) urine volume $<0.5 \mathrm{~mL} / \mathrm{kg}$.h and time of duration $>6$ hours. ${ }^{6}$ Recovery standard within seven days after AKI: (1) complete recovery: the last time $\mathrm{SCr}$ within seven days was $<1.2$ times of baseline; (2) partial recovery: the last time $\mathrm{SCr}$ in seven days was $>1.2$ times of baseline and $<1.5$ times; (3) no recovery: the last time $\mathrm{SCr}$ in seven days was $\geq 1.5$ times of the diagnosis, or continued renal replacement therapy; (4) non-detection: no duplicate detection was carried out within seven days after diagnosing $\mathrm{AKI}^{7}$ Its evaluation means include the SCr level, glomerular filtration rate, changes in kidney injury, relevant biological indicators related to kidney function recovery, and (or) other methods to evaluate kidney reserve function and kidney blood perfusion. Therefore, the present study proposes to review the application situation and research progress of the 
recoverability evaluation of $\mathrm{AKI}$ and $\mathrm{AKD}$ patient kidney function from three aspects: regular monitoring indicators, biomarkers, and imaging examination.

\section{Regular Monitoring Indicator}

At present, the conventional indexes to inspect kidney functions in clinic mainly include creatinine, urine volume, glomerular filtration rate, etc. Since it is not suitable to adopt inulin, ${ }^{51} \mathrm{Cr}$-EDTA, ${ }^{99} \mathrm{TmDTPA}$, or iodohydrin to measure the glomerular filtration rate for critically ill patients received by the intensive care unit (ICU), the kidney function evaluation for critically ill patients was limited to serum creatinine and urine volume. Creatinine and urine volume are influenced by various reasons, such as gender, age, weight, nutrient status and so on. ${ }^{8}$ When $\mathrm{SCr}$ increases and exceeds the normal range, and the glomerular filtration rate decreases by $>50 \%$, this indicates that renal parenchyma damage actually occurred. The creatinine concentration is influenced by body fluid volume change. If the volume is overloaded, the AKI diagnosis may be postponed. ${ }^{9}$ Furthermore, the urine volume is influenced by the volume state, fluid ADI, diuretic and other factors, and the urine volume decrease may be the reaction of decrease of normal renal perfusion pressure or kidney injury. Therefore, AKI diagnosis, with creatinine and urine volume as the measurement criteria, lacks the ideal sensibility and specificity. ${ }^{10}$

\section{Biomarker}

At present, the biomarkers to evaluate kidney function damage in clinic mainly include the following: neutrophil gelatinase-associated lipocalin (NGAL), kidney injury molecule 1 (KIM-1), interleukin-18 (IL-18), cystatin $\mathrm{C}$ CysC, urinary total protein, matrix metalloproteinase-9, retinol conjugated protein, etc. However, different biomarkers have different advantages and disadvantages. Various previous studies have indicated that the increase in biomarkers, such as IL-18, KIM-1, NGAL and CysC, occurs earlier, when compared to creatinine, urea nitrogen and urine volume, and the combination of these is expected to increase the sensibility of AKI diagnosis. ${ }^{6,11}$ Although it remains unclear whether there is a factor to stimulate the continuous increase in biomarker level when the kidney injury biomarker level is continuously increased at present, the above biomarker level increase is still considered as a signal of continuous kidney injury. The decrease in kidney injury biomarkers has been used to predict the AKI and AKI recovery after a kidney transplant. These biomarkers did not decline, which may reflect the risk of AKI progressing to $\mathrm{AKD}$ or $\mathrm{CKD}$, in addition to indicating "irrecoverable". ${ }^{12}$ Common biomarkers that can be used to evaluate AKI recoverability would be respectively introduced.

\section{NGAL}

NGAL is an apolipoprotein associated with the neutrophil gelatinase, and it is mainly expressed in the epithelial cells of renal proximal tubular cells. After kidney injury, the main sources of urinary NGAL are the renal collecting duct and the ascending branch of the medullary loop. ${ }^{13}$ Increased NGAL could be detected in early (within two hours) urine of AKI. NGAL is the most valuable biomarker for the diagnosis of AKI, and could be used to predict the severity and duration of AKI. The specificity and sensitivity of NGAL is $81 \%$ and $68 \%$, respectively. ${ }^{14}$ NGAL detected in urine or serum can give a good prediction for kidney injury, but is liable to be influenced by AKI complication, such as sepsis, chronic renal failure, malignant tumor, inflammatory diseases, etc. ${ }^{15}$ Urine NGAL decreases oxidative stress by adjusting the iron concentration in cells, but this has little significance in AKI patient clinical prognosis as the kidney tissue damage maker. ${ }^{16}$ In present clinical practice, there is no unified NGAL threshold prediction and evaluation for AKI.

\section{KIM-I}

KIM-1 is a I transmembrane glycoprotein, which is mainly expressed on the surface of $\mathrm{T}$ cells, but has little expression on healthy kidneys or urine. ${ }^{17}$ KIM-1 expression in kidney tubular epithelial cells significantly increase after kidney injury. ${ }^{18}$ Han et al ${ }^{19}$ conducted a prospective study on clinical cases, and revealed that KIM-1 was detected in urine after 12 hours of kidney ischemic injury. The increase in level is apparently higher than other types of kidney injury. When KIM-1 increases by $1 \mathrm{U}$, the probability of acute kidney failure would increase by 12 times. Hence, KIM-1 can not only be used for the early diagnosis of AKI, its value can be used for evaluating and monitoring the disease status. Urine KIM-1 level is significantly higher in acute tubular necrosis patients than in patients with chronic kidney disease and contrast nephropathy. ${ }^{20}$ For the operation of patients, the area under the curve (AUC) values of AKI in children and adult urine KIM-1 is 0.83 and 0.78 , respectively. ${ }^{21} \mathrm{KIM}-1$ in urine can reflect the damage of proximal renal tubular epithelial cells. In acute kidney injury patients, urine KIM-1 begins to 
increase after two hours of injury, and reaches a peak value within 48 hours. Hence, urine KIM-1 determination is an important biological marker to diagnose AKI, and evaluate the disease status and prognosis.

\section{IL- 18}

IL-18 is the proinflammatory factor secreted by kidney tubular cells, macrophages and other antigen-presenting cells. IL-18 kidney tubular cells and macrophages synthesize the IL-18 inactive precursor, and is cracked by cysteine-aspartic protease- 1 or released in blood after the secretion of monocytes/macrophages. ${ }^{22}$ Cleaved IL-18 plays an inflammatory role through receptor and receptor heterodimer signaling pathways. During the ischemic injury, sepsis and malignant tumor period, IL-18 is released in the urine. Then, the urine IL-18 begins to increase within six hours of kidney injury, and reaches a peak within 12-18 hours. In a study, within the first three days after cardiac surgery, urine IL-18 is the best predictor of AKI progression or stage I patient AKI death. $^{23}$ One meta-analysis revealed that the AUC of AKI predicted by the urine IL-18 level was 0.77 . This indicates that IL-18 has a moderate or above value in the early diagnosis $\mathrm{AKI}{ }^{24}$ Furthermore, it is found that urine IL-18 level in patients with cirrhosis can be used to distinguish ischemic acute tubular necrosis and other types of renal damage, and predict the short-term mortality in such patients. $^{25}$ The increase of IL-18 in urine is liable to be influenced by sepsis, inflammation, lung injury, heart failure, immune injury and other factors, causing this to poorly predict the long-term prognosis of AKI patients. ${ }^{26}$ However, IL-18 therapy may be used as an AKI therapeutic method in the future.

\section{CysC}

CysC is mainly generated by karyotes, and this can almost be completely filtered by glomeruli, and finally completely reabsorbed in proximal tubules. ${ }^{27}$ Hence, there is no CysC in urine. $\mathrm{Cys} C$ is not influenced by gender, age and muscle content. Furthermore, its distribution volume is $1 / 3$ of creatinine, and it could more quickly reflect the kidney function injury, when compared to serum creatinine. An increase in urine $\mathrm{CysC}$ suggests that there is renal kidney injury. Therefore, $\mathrm{CysC}$ has certain advantages in the early diagnosis AKI and prognosis evaluation. ${ }^{28}$ A study revealed that in acute respiratory distress in premature newborns, when the CysC threshold is $>1.3 \mathrm{mg} / \mathrm{L}$, the sensitivity and specificity for AKI diagnosis is $92.3 \%$ and $96 \%$, respectively. This can earlier predict the occurrence of AKI, when compared to creatinine and the glomerular filtration rate. $^{29}$ For the early diagnosis of AKI, CysC can diagnose AKI at 24-48 hours earlier, when compared to $\mathrm{SCr}^{30}$ However, for serious kidney injury, since the glomerular filtration rate is significantly decreased, serum CysC has no advantages, when compared to $\mathrm{SCr}^{31}$

\section{Human Cartilage Glycoprotein 39}

White cartilage glycoprotein 39 is a glycoprotein involved in inflammation, cell protection and repair, and is produced by a variety of cells, which include kidney macrophages. The high urine level of human cartilage glycoprotein-39 in dead kidney donors is not only correlated to $\mathrm{AKI}$, but also correlated to the improvement of graft function and the glomerular filtration rate at six months. This may indicate that the increase of this marker may represent the beginning of an effective repair. ${ }^{12}$ Hence, human cartilage glycoprotein 39 is a potential biomarker that may be used to predict the severity and recovery of AKI.

In summary, there is no consensus on when to use biomarkers to predict renal recovery time. The reason is correlated to the confounding factors (sepsis, fluid status, etc.) and severity of $\mathrm{AKI}$ or $\mathrm{AKD}$, and the determination on whether to accept renal replacement therapy. ${ }^{14}$ Unlike the use of troponins for confirmation of myocardial infarction, identification of a single marker for AKI seems unlikely.

\section{Imaging Methods}

Since kidney function is significantly correlated with the blood perfusion of renal parenchyma, the dynamic detection of renal blood perfusion may sensitively reflect the changes in kidney function. At present, the common imaging methods for evaluating renal function through clinical application are radionuclide renal dynamic imaging, spiral computed tomography (CT) enhancement scan, magnetic resonance imaging (MRI), and ultrasound.

\section{Radionuclide Renal Dynamic Imaging}

Radionuclide renal dynamic imaging is the clinical standard of determining the glomerular filtration rate at present, with the classic method of 99mTc-DTPA renal dynamic imaging, Gates. The test method is performed to conduct the radioactive counting of the drug in the kidneys after the intravenous injection of $99 \mathrm{mTcDTPA}$ 
for a period of time, in order to calculate the glomerular filtration rate and the corresponding imaging of urinary system. ${ }^{32}$ This may provide the information on the morphology of double kidneys, the function of the renal parenchyma, and the unobstructed urinary tract and renal blood perfusion, using the 99mTc-DTPA renal dynamic imaging Gates method. At present, the method applied is the imaging method for obtaining the split-renal function for non-invasive quantification, and this is used to evaluate the severity and prognosis of renal disease in clinic, and the change in illness before and after treatment, and guide the dialysis treatment of patients with renal failure, or evaluate the timing of the renal transplantation. ${ }^{23}$ However, when the glomerular filtration rate of the patient is decreases to a certain degree, the determination results with this method would no longer be accurate, which may have a certain effect on the evaluation of kidney function and guidance of clinical treatment. ${ }^{33,34}$ Hence, there are many disputes for the accuracy of renal dynamic imaging through the Gates method.

\section{Spiral CT Enhancement Scan}

Iopromide or iohexol is the commonly used CT contrast agent, which would not be secreted or reabsorbed in the kidney tubule. ${ }^{35}$ This may be used as a tracer agent to determine the kidney function. There is a good linear relation between the concentration of renal CT contrast agent and $\mathrm{CT}$ attenuation value. Therefore, the product of kidney volume and $\mathrm{CT}$ enhancement value may be used for evaluating kidney function. ${ }^{36}$ Hence, spiral CT is a reliable tool to determine whether the contrast agent is filtered by the kidney. Renal blood perfusion and renal filtration effect the $\mathrm{CT}$ enhancement at different stages, and have different effects on renal CT value. ${ }^{35,36}$ Therefore, contrast media can cause renal injury, which limits the application of enhanced CT.. The accuracy of kidney function by the CT enhancement scan remains to be improved.

\section{Magnetic Resonance Imaging}

MRI techniques that are applied more in the evaluation of kidney function are diffusion weighted imaging (DWI) and diffusion tensor imaging (DTI). The principles are to reflect the changes in the pathophysiology of renal tissues through the measurement of diffusion of water molecules and tissue perfusion in the kidney. DTI was developed and deepened from DWI, which has quantified the directional preference of water molecule diffusion. The movement characteristics of water molecules can be expressed by the apparent diffusion coefficient (ADC). ADC combines the parameters of dispersion and perfusion to reflect the diffusibility in the whole tissue. The diffusion characteristics of water molecules in the kidney may reflect the kidney function. Some previous studies speculate that the ADC value may directly reflect the damage degree of kidney function, based on the correlation between the $\mathrm{ADC}$ value and renal function indicator. ${ }^{37}$ However, $\mathrm{ADC}$ is a semi-quantitative indicator, and can indirectly reflect the kidney function. A large number of studies are still needed to further confirm the sensitivity and specificity of renal function impairment diagnosed by ADC.

\section{Evaluation of Kidney Function by Two-Dimensional Ultrasound}

Although the information of renal structure and renal blood perfusion can be obtained with the imaging examination methods above, this is not suitable for evaluating the kidney function of patients with kidney injury due to limitations, such as the renal toxicity of contrast agent, expensive examination price, radiation failure of point-of-care, dynamic and bedside testing, etc. Point of care ultrasound has rapidly been developed in recent years. At present, the main ultrasonic methods for evaluating renal perfusion are twodimensional ultrasound and Doppler ultrasound resistance index determination with ultrasound contrast. For twodimensional ultrasound, the normal kidney is oval, the renal parenchyma has a low-level echo, which is lower than that of the liver and spleen, under this kind of ultrasound, and the renal sinus presents a high-level echo. The long diameter of the normal kidney was 9-12 cm, the transverse diameter was 5-7 cm, and the thickness was 4-6 cm. The thickness of the renal parenchyma was $1.4-1.8 \mathrm{~cm}$. The renal long diameter was the most useful parameter to identify acute and chronic renal diseases, and evaluate the kidney function in the two-dimensional measurement parameters of kidneys. At the same time, twodimensional ultrasound may screen the renal insufficiency that resulted from the cause of obstruction. The renal parenchyma is thickened and kidney volume is increased at different degrees in the case of acute renal insufficiency. ${ }^{38}$ The acute renal insufficiency, which resulted from the renal venous thrombosis, acute tubular necrosis and acute interstitial nephritis, may lead to the increase of kidney volume. Among the patients who have renal failure that resulted from chronic glomerulonephritis, with the progression of disease, the kidney volume is gradually reduced, the cortex echo is 
enhanced, and the corticomedullary differentiation is vague. $^{39}$

\section{Doppler Ultrasound Kidney Resistance Index}

Renal blood flow is mainly determined by kidney vessel (including the afferent arteriole, efferent arteriole and interlobar artery) resistance. The changes in renal blood perfusion can be evaluated half-quantitatively through the positioning of the vessels above. Lerolle reported that for critical patients diagnosed with AKI, there was a significant correlation between the increase in resistant index (RI) and occurrence of AKI through the repeated measurement of RI of the interlobar artery or arcuate artery. ${ }^{40}$ Renal RI has no normal value at present. In general, a healthy person's RI is approximately 0.6 , with a high limit of $0.7 .^{41}$ Radermacher found that the changes in RI of patients after the renal transplantation correlated with the prognosis. ${ }^{42}$ Some studies have revealed that RI obviously increased at the AKI oliguria stage and migratory stage, and that this was more than 0.7 in general. Furthermore, 2-3 times of monitoring was conducted every week at the AKI oliguria stage and recovery stage. If RI processivity was reduced, the prognosis would be better, while if RI continued to grow or had no significant decline, this suggested that the prognosis was poorer. ${ }^{43}$ Measuring RRI immediately after major surgery and using a cut-off value $\geq 0.715$ may provide an excellent tool in the detection of the onset of AKI. ${ }^{44}$ However, RI is closely related to cardiac function, vascular elasticity and renal interstitial condition, and is not a good indicator of renal perfusion, ${ }^{45}$ the measurement of kidney RI by ultrasound are affected by factors, such as the patient's breathing activity, body position, arterial compliance and intraabdominal pressure, etc. Hence, ultrasound cannot accurately quantify the renal blood flow velocity. In recent years, studies on the use of renal RI to determine and evaluate AKI have been steadily decreasing. The investigators consider that for patients with AKI that resulted from different diseases, more studies are needed to evaluate the value of renal RI to AKI in evaluating the critical degree and predicting the prognosis.

\section{Ultrasound Contrast}

Among AKI patients, the changes in renal blood perfusion was significant. ${ }^{44}$ Furthermore, $90 \%$ of the renal blood perfusion is concentrated on the renal cortex, and all or the local renal blood perfusion of the renal cortex have various degrees of changes at the early stage of renal injury. ${ }^{45}$ The microbubble contrast agent completely cycles in the blood vessel after being injected into the peripheral vein, which belongs to the hemodynamic change, and is similar to red blood cell (RBC) movement. The renal ultrasound contrast imaging successively revealed that the renal-artery-renal cortex-renal medulla was rapidly enhanced. Hence, the early diagnosis of the renal lesion and renal perfusion should be conducted using the ultrasonic contrast value and time intensity curve (TIC) at different time points. In a study on the ultrasound contrast of an AKI rabbit model induced by cisplatinum, ${ }^{46} \mathrm{SCr}$ was found earlier than AKI by the ultrasound contrast, and the severity was evaluated. Cao et $\mathrm{al}^{47}$ reported that the renal ultrasound contrast may dynamically and non-invasively monitor the renal ischemic reperfusion injury and early predict the progression of AKI-CKD. The clinical application of the ultrasound contrast is increasing, but there is still has no accepted renal perfusion standard. ${ }^{48}$ This limits quantitative renal blood perfusion to some extent.

Even though the biomarker may judge the severity of kidney injury and suggest the patient's prognosis to some extent, there is still no standard for diagnosing kidney injury at present. This category is likely to be the diagnosis and evaluation standards for kidney injury when the new biomarker is studied at the same time. For the imaging, bedside ultrasound is a good method for the early evaluation of AKI and renal perfusion, and prediction of the patient's prognosis. The dynamic changes in RI may have a certain value to the prognosis of AKI patients. Although there have been few studies, the contrastenhanced ultrasound technique has exhibited the characteristics of real-time evaluation, no damage and high accuracy to the renal perfusion. In addition, this allows for the more accurate evaluation of the situation of the local and overall kidney of AKI patients, in combination of the targeted drug and contrast agent, using the microbubble technique.

In conclusion, although there are many limitations and disadvantages for evaluating the kidney function of critical patients by creatinine and urine volume, creatinine and urine volume are still the important indicators for diagnosing and evaluating kidney injury.

\section{Disclosure}

The authors declare that there are no conflicts of interest. 


\section{References}

1. Marx D, Metzger J, Pejchinovski M, et al. Proteomics and metabolomics for AKI diagnosis. Semin Nephrol. 2018;38(1):63-87. doi:10.1016/j.semnephrol.2017.09.007

2. Kiryluk K, Bomback AS, Cheng YL, et al. Precision medicine for acute kidney injury (AKI): redefining AKI by agnostic kidney tissue interrogation and genetics. Semin Nephrol. 2018;38(1):40-51. doi:10.1016/j.semnephrol.2017.09.006

3. Gammelager H, Christiansen CF, Johansen MB, Tønnesen E, Jespersen B, Sørensen HT. One-year mortality among Danish intensive care patients with acute kidney injury: a cohort study. Crit Care. 2012;16(4):R124. doi:10.1186/cc11420

4. Beker BM, Corleto MG, Fieiras C, Musso CG. Novel acute kidney injury biomarkers: their characteristics, utility and concerns. Int Urol Nephrol. 2018;50(4):705-713. doi:10.1007/s11255-017-1781-x

5. Merchant ML, Brier ME, Slaughter MS, Klein JB, McLeish KR. Biomarker enhanced risk prediction for development of AKI after cardiac surgery. BMC Nephrol. 2018;19(1):102. doi:10.1186/s12882018-0902-9

6. Matsuura R, Komaru Y, Miyamoto Y, et al. Response to different furosemide doses predicts AKI progression in ICU patients with elevated plasma NGAL levels. Ann Intensive Care. 2018;8(1):8. doi:10.1186/s13613-018-0355-0

7. Chawla LS, Bellomo R, Bihorac A, et al.; Acute Disease Quality Initiative Workgroup. Acute kidney disease and renal recovery: consensus report of the acute disease quality initiative (ADQI) 16 workgroup. Nat Rev Nephrol. 2017;13(4):241-257. doi:10.1038/ nrneph.2017.2

8. Ostermann M, Kashani K, Forni LG. The two sides of creatinine: both as bad as each other? $J$ Thorac Dis. 2016;8(7):E628-630. doi:10.21037/jtd.2016.05.36

9. Schrezenmeier EV, Barasch J, Budde K, Westhoff T, Schmidt-Ott KM. Biomarkers in acute kidney injury - pathophysiological basis and clinical performance. Acta Physiol. 2017;219(3):554-572. doi:10.1111/apha.12764

10. Albert C, Albert A, Kube J, et al. Urinary biomarkers may provide prognostic information for subclinical acute kidney injury after cardiac surgery. J Thorac Cardiovasc Surg. 2018;155(6):2441-2452. e13. doi:10.1016/j.jtcvs.2017.12.056

11. Vysakh A, Raji NR, Suma D, Jayesh K, Jyothis M, Latha MS. Role of antioxidant defence, renal toxicity markers and inflammatory cascade in disease progression of acute pyelonephritis in experimental rat model. Microb Pathog. 2017;109(12):189-194. doi:10.1016/j. micpath.2017.05.047

12. Endre ZH. Assessing renal recovery after acute kidney injury: can biomarkers help? Nephron. 2018;140(2):86-89. doi:10.1159/0004 92290

13. Singer E, Markó L, Paragas N, et al. Neutrophil gelatinase-associated lipocalin: pathophysiology and clinical applications. Acta Physiol. 2013;207(4):663-672. doi:10.1111/apha.12054

14. Nickolas TL, Schmidt-Ott KM, Canetta P, et al. Diagnostic and prognostic stratification in the emergency department using urinary biomarkers of nephron damage: a multicenter prospective cohort study. J Am Coll Cardiol. 2012;59(3):246-255. doi:10.1016/j.jacc.20 11.10 .854

15. Di Somma S, Magrini L, De Berardinis B, et al. Additive value of blood neutrophil gelatinase-associated lipocalin to clinical judgement in acute kidney injury diagnosis and mortality prediction in patients hospitalized from the emergency department. Crit Care. 2013;17(1): R29. doi: $10.1186 / \mathrm{cc} 12510$

16. Biernawska J, Bober J, Kotfis K, Bogacka A, Barnik E, Żukowski M. Cardiac surgery related cardio-renal syndrome assessed by conventional and novel biomarkers under or overestimated diagnosis? Arch Med Sci. 2017;13(5):1111-1120. doi:10.5114/aoms.2017.69328
17. Obermuller N, Geiger H, Weipert C, Urbschat A. Current developments in early diagnosis of acute kidney injury. Int Urol Nephrol. 2014;46(1):1-7. doi:10.1007/s11255-013-0448-5

18. Jin Y, Shao X, Sun B, Miao C, Li Z, Shi Y. Urinary kidney injury molecule-1 as an early diagnostic biomarker of obstructive acute kidney injury and development of a rapid detection method. Med Rep. 2017;15(3):1229-1235. doi:10.3892/mmr.2017.6103

19. Han WK, Bailly V, Abichandani R, Thadhani R, Bonventre JV. Kidney injury molecule-1 (KIM-1): a novel biomarker for human renal proximal tubule injury. Kidney Int. 2002;62(1):237-244. doi:10.1046/j.1523-1755.2002.00433.x

20. Kokkoris S, Pipili C, Grapsa E, Kyprianou T, Nanas S. Novel biomarkers of acute kidney injury in the general adult ICU: a review. Ren Fail. 2013;35(4):579-591. doi:10.3109/0886022X.2013.773835

21. Slocum JL, Heung M, Pennathur S. Marking renal injury: can we move beyond serum creatinine? Transl Res. 2012;159(4):277-289. doi:10.1016/j.trsl.2012.01.014

22. Choudhary A, Basu S, Dey SK, Rout JK, Das RK, Dey RK. Association and prognostic value of serum Cystatin C, IL-18 and uric acid in urological patients with acute kidney injury. Clinica Chimica Acta. 2018;482:144-148. doi:10.1016/j.cca.2018.04.005

23. Mishra OP, Rai AK, Srivastava P, et al. Predictive ability of urinary biomarkers for outcome in children with acute kidney injury. Pediatric Nephrol. 2017;32(3):521-527. doi:10.1007/s00467-016-3445-y

24. Lin X, Yuan J, Zhao Y, Zha Y. Urine interleukin-18 in prediction of acute kidney injury: a systemic review and meta-analysis. $J$ Nephrol. 2015;28(1):7-16. doi:10.1007/s40620-014-0113-9

25. Puthumana J, Ariza X, Belcher JM, Graupera I, Ginès P, Parikh CR. Urine interleukin 18 and lipocalin 2 are biomarkers of acute tubular necrosis in patients with cirrhosis: a systematic review and metaanalysis. Clin Gastroenterol Hepatol. 2017;15(7):1003-1013. doi:10.1016/j.cgh.2016.11.035

26. Nisula S, Yang R, Poukkanen M, et al.; FINNAKI Study Group. Predictive value of urine inter leukin-18 in the evolution and outcome of acute kidney injury in critically ill adult patients. $\mathrm{Br} J$ Anaesth. 2015;114(3):460-468. doi:10.1093/bja/aeu382

27. de Geus HR, Betjes MG, Bakker J. Biomarkers for the prediction of acute kidney injury: a narrative review on current status and future challenges. Clin Kidney J. 2012;5(2):102-108. doi:10.1093/ckj/sfs008

28. Hu Y, Liu H, Du L, Wan J, Li X. Serum cystatin C predicts AKI and the prognosis of patients in coronary care unit: a prospective, observational study. Kidney Blood Press Res. 2017;42(6):961-973. doi:10.1159/000485341

29. El-Gammacy TM, Shinkar DM, Mohamed NR, Al-Halag AR. Serum cystatin $\mathrm{C}$ as an early predictor of acute kidney injury in preterm neonates with respiratory distress syndrome. Scand J Clin Lab Invest. 2018;78(5):352-357. doi:10.1080/00365513.2018.1472803

30. Leem AY, Park MS, Park BH, et al. Value of serum cystatin $\mathrm{C}$ measurement in the diagnosis of sepsis-induced kidney injury and prediction of renal function recovery. Yonsei Med J. 2017;58 (3):604-612. doi:10.3349/ymj.2017.58.3.604

31. Fouad M, Boraie M. Cystatin C as an early marker of acute kidney injury and predictor of mortality in the intensive care unit after acute myocardial infarction. Arab J Nephrol Transplant. 2013;6(1):21-26.

32. Gates GF. Glomerular filtration rate: estimation from fractional renal accumulation of 99mTc-DTPA (stannous). Am J Roentgenol. 1982;138(3):565-570. doi:10.2214/ajr.138.3.565

33. Morine Y, Enkhbold C, Imura S, et al. Accurate estimation of functional liver volume using Gd-EOB-DTPA MRI compared to MDCT/ 99mTc-SPECT fusion imaging. Anticancer Res. 2017;37(10):56 93-5700. doi:10.21873/anticanres.12006

34. Assadi M, Eftekhari M, Hozhabrosadati M, et al. Comparison of methods for determination of glomerular filtration rate: low- and high-dose Tc-99m-DTPA renography, predicted creatinine clearance method, and plasma sample method. Int Urol Nephrol. 2008;40 (4):1059-1065. doi:10.1007/s11255-008-9446-4 
35. Yuan X, Zhang J, Tang K, et al. Determination of glomerular filtration rate with $\mathrm{CT}$ measurement of renal clearance of iodinated contrast material versus ${ }^{99 \mathrm{~m}}$ Tc-DTPA dynamic imaging "gates" method: a validation study in asymmetrical renal disease. Radiology. 2016;282 (2):552-560. doi:10.1148/radiol.2016160425

36. You S, Ma X, Zhang C, et al. Determination of single-kidney glomerular filtration rate (GFR) with CT urography versus renal dynamic imaging Gates method. Eur Radiol. 2018;28(3):10 77-1084. doi:10.1007/s00330-017-5061-z

37. Xu X, Fang W, Ling H, Chai W, Chen K. Diffusion-weighted MR imaging of kidneys in patients with chronic kidney disease in patients with chronic kidney disease: initial study. Eur Radiol. 2010;20 (4):978-983. doi:10.1007/s00330-009-1619-8

38. Dayal M, Gamanagatti S, Kumar A. Imaging in renal trauma. World J Radiol. 2013;5(8):275-284. doi:10.4329/wjr.v5.i8.275

39. Rumack CM, Wilson SR, Charboneau JW. Diagnostic Ultrasound. 3rd ed. St. Louis: Elsevier Health Sciences; 2005.

40. Lerolle N, Guérot E, Faisy C, Bornstain C, Diehl JL, Fagon JY. Renal failure in septic shock: predictive value of Doppler-based renal arterial resistive index. Intensive Care Med. 2006;32(10):1553-1559. doi:10.1007/s00134-006-0360-x

41. Le Dorze M, Bouglé A, Deruddre S, Duranteau J. Renal Doppler ultrasound: a new tool to assess renal perfusion in critical illness. Shock. 2012;37(4):360-365. doi:10.1097/SHK.0b013e3182467156
42. Radermacher J, Mengel M, Ellis S, et al. The renal arterial resistance index and renal allograft survival. $N$ Engl J Med. 2003;349 (2):115-124. doi:10.1056/NEJMoa022602

43. Craig WD, Wagner BJ, Travis MD. Pyelonephritis: radiologic-pathologic review. Radiographics. 2008;28(1):255-277. doi:10.1148/rg.281075171

44. Wu H, Liu K, Darko IN, et al. Predictive value of renal resistive index for the onset of acute kidney injury and its non-recovery: a systematic review and meta-analysis. Clin Nephrol. 2020;93 (4):172-186. doi:10.5414/CN109979

45. Di Nicolò P, Granata A. Renal resistive index: not only kidney. Clin Exp Nephrol. 2017;21(3):359-366. doi:10.1007/s10157-016-1323-3

46. Harrois A, Grillot N, Figueiredo S, Duranteau J. Acute kidney injury is associated with a decrease in cortical renal perfusion during septic shock. Critical Care. 2018;22(1):161. doi:10.1186/ s13054-018-2067-0

47. Cao W, Cui S, Yang L, et al. Contrast-enhanced ultrasound for assessing renal perfusion impairment and predicting acute kidney injury to chronic kidney disease progression. Antioxid RedoxSignal. 2017;27(17):1397-1411. doi:10.1089/ars.2017.7006

48. Wang L, Mohan C. Contrast-enhanced ultrasound: a promising method for renal microvascular perfusion evaluation. J Transl Int Med. 2016;4(3):104-108. doi:10.1515/jtim-2016-0033
International Journal of General Medicine

\section{Publish your work in this journal}

The International Journal of General Medicine is an international, peer-reviewed open-access journal that focuses on general and internal medicine, pathogenesis, epidemiology, diagnosis, monitoring and treatment protocols. The journal is characterized by the rapid reporting of reviews, original research and clinical studies
Dovepress

across all disease areas. The manuscript management system is completely online and includes a very quick and fair peer-review system, which is all easy to use. Visit http://www.dovepress.com/ testimonials.php to read real quotes from published authors. 